\title{
The Early Use of Captopril in Acute Myocardial Infarction
}

\author{
Hafed I Hussein DM* \\ Ramadi General Teaching Hospital, Al-Anbar Province, Iraq
}

Submission: April 04, 2018; Published: July 13, 2018

*Corresponding author: Hafed I Hussein DM, Ramadi General Teaching Hospital, Al-Anbar Province, Iraq, Email: aldulaimihafidh61@gmail.com

Abstract

A prospective study on the effect of early use of captopril (ACE inhibitors) on the management of patients with acute myocardial infarction was carried out during the period from April 1996 to October 1996, in the coronary care unit (CCU) of Saddam General Hospital in Ramadi.

Two groups were taken, the first sample of 31 patients (22 males and 9 females) received captopril, and another sample of 29 patients (18 males and 11 females) received the conventional therapy.

The study showed that on both short and long term management, captopril was found to reduce incidence of mortality as well as left ventricular dysfunction. All results obtained were upon sequential clinical findings, ECG, chest X-ray, and Echocardiography.

Keywords: Captopril; MI; Echocardiography

\section{Introduction}

Cardiac performance is determined by three important variables: preload, the force of cardiac contraction, and afterload [1]. Preload refers to the volume of blood stretching the left ventricular just prior to contraction, or the left ventricular and diastolic volume (LVEDV), normal value 120-130ml [2]. Afterload refers to the resistance or impedance against which the heart must contract. Clinically primary determinant is the resistance, (PVR). The inadequate ventricular systolic function may be due to loss of functioning muscle and/or depressed contractility of muscle, as in ischaemic heart diseases [3]. Myocardial infarction and loss of a functioning left ventricular effectively place the remainder of the muscle under a volume load, even in the absence of ventricular aneurysm where the effect is more widely accepted. Probably more important ischaemic consistently results in depression of left ventricular function. The strongest indicator of poor prognosis of myocardial infarction is deteriorating ventricular function. The possibility of preventing such deterioration through very early intervention with angiotensin-converting-enzyme (ACE) inhibitors.

Captopril is the first member of an exciting new group of compounds ACE inhibitors to become widely available for clinical use [3]. Captopril inhibits the enzyme that converts the inactive angiotensin I to the potent vasoconstrictor angiotensis II. It also has some further action in lowering blood pressure by decreasing the breakdown of bradykinin. Captopril reduces aldosterone secretion and can thus raise plasma potassium concentration. Approximately $35 \%$ of a dose of captopril is eliminated unchanged in the urine [1]. Therefore, it must be used with more caution in renal insufficiency.

\section{Patients and Methods}

A sample of 31 patients with acute myocardial infarction admitted to the CCU in Saddam General Hospital in Ramadi was considered in this study. The sample contained 22 males (71\%) and 9 females (29\%). Collection of items of our sample was done during the period April to October 1996. Another sample of 29 patients, 18 males (62\%) and 11 females (38\%) were also collected during the same period and in the same unit.

Patients of both groups were found to have acute myocardial infarction, this was based on clinical findings (i.e., typical ischaemic chest pain lasting more than one hour), ECG changes (new appearance of $\mathrm{Q}$ wave with evolutionary ST \& $\mathrm{T}$ wave changes), and rise in the enzymatic level.

Two dimensional echocardiography was done at frequent visits to assess segmental left ventricular function. Ejection fraction was calculated as the difference between n-diastolic and n-systolic volume as a percentage of diastolic volume. Normal values from $39-69 \%$. The lower the percentage, the more is the 


\section{Journal of Cardiology \& Cardiovascular Therapy}

dysfunction. Assessment of left ventricular wall motion was based on two dimensional tomograms a kinetic segment wall motion of left ventricular wall also studied.

The groups were managed according to two main therapy regimens. The first sample (31 patients) was treated by the use of captopril on the basis that patients received a dose of $6.25-12.5 \mathrm{mg}$ once daily, started 3-10 days after MI. The dose was gradually increased up to $50 \mathrm{mg}$ three times daily. Just before starting the treatment, an echocardiography was done for all patients.

Serum creatinine and blood urea were also done for patients of this sample to exclude patients with renal dysfunction. Patients with hypotension as well as patients received already ACE inhibitors were also excluded from the study. Patients of the other sample were put on a conventional therapy (analgesic, vasodialator, and oxygen, ..., etc.). Patients in both groups were followed for a short (10 days in hospital) and long term (up to six months after discharging from hospital).

\section{Results}

The type of MI as found on the bases of ECG findings for the two groups is shown on Table 1.
Table 1: The type of MI determined on the bases of ECG findings.

\begin{tabular}{|c|c|c|c|c|}
\hline \multirow{2}{*}{ Type of MI } & \multicolumn{2}{|c|}{ Captopril } & \multicolumn{2}{c|}{ Control } \\
\cline { 2 - 5 } & Male & Female & Male & Female \\
\hline Anterio-lateral \& septal & 8 & - & 4 & 1 \\
\hline Anterioseptal & 4 & 1 & 3 & 2 \\
\hline Anteriolateral & 5 & 3 & 3 & 5 \\
\hline Inferior & 3 & 3 & 3 & 2 \\
\hline Sub-endocardial & 2 & 1 & 4 & - \\
\hline Posterior & - & 1 & 1 & 1 \\
\hline Total & 22 & 9 & 18 & 11 \\
\hline
\end{tabular}

It is clear from this table that the incidence of extensive MI (anteriolateral and anterioseptal) and anteriolateral in both groups is the highest among all other types of MI in our study, $50 \%$ and $54 \%$ respectively for the sample of captopril and control.

On the long and short term of treatment, patients of both groups were observed for their prospective health status. After six months of following-up, Table 2 shows the changes from LV dysfunction to normal LV function and vice versa for both groups of therapies, number of dead patients is also presented in this table.

Table 2: Type of therapy and state of LV function in both groups of patients explained by the ECG findings and Echocardiography after 6 months of follow-up.

\begin{tabular}{|c|c|c|c|c|c|c|c|}
\hline \multirow{3}{*}{ Therapy } & \multicolumn{2}{|c|}{ Week 1} & \multicolumn{4}{|c|}{6 Months } & \multirow{3}{*}{ Dead } \\
\hline & \multirow[t]{2}{*}{ State of LV Func. } & \multirow[t]{2}{*}{ No. } & \multicolumn{2}{|c|}{ LV Dysfunc. } & \multicolumn{2}{|c|}{ Normal LV Func. } & \\
\hline & & & No. & $\%$ & No. & $\%$ & \\
\hline \multirow{3}{*}{ Captopril } & Total & 31 & & & & & \\
\hline & LV dysfunction & 23 & 8 & 34.78 & 14 & 60.87 & 1 \\
\hline & Normal LV function & 8 & 1 & 12.5 & 7 & 87.50 & - \\
\hline \multirow{3}{*}{ conventional } & Total & 29 & & & & & \\
\hline & LV dysfunction & 15 & 10 & 66.67 & 3 & 20.00 & 2 \\
\hline & Normal LV function & 14 & 5 & 35.71 & 8 & 57.14 & 1 \\
\hline
\end{tabular}

Table 3: Type of LV dysfunction showed by two-dimensional echocardiography.

\begin{tabular}{|c|c|c|c|c|}
\hline \multirow{2}{*}{ Type of LV Dysfunction } & \multicolumn{2}{|c|}{ Captopril } & \multicolumn{2}{c|}{ Conventional } \\
\cline { 2 - 5 } & No. & $\%$ & No. & $\%$ \\
\hline Ejection fraction below 39\% & 3 & 37.5 & 4 & 40 \\
\hline Assessment of wall function & 2 & 25 & 3 & 30 \\
$-\quad$ akinesis & 3 & 37.5 & 3 & 30 \\
$-\quad$ dyskinesis & & & & \\
\hline LV size: & 21 & 67.74 & 11 & 37.93 \\
1. EDD (Normal $5.7 \mathrm{~cm})$ & 21 & 67.74 & 11 & 37.93 \\
2. ESD (Normal $4.0 \mathrm{~cm})$ & & & & \\
\hline
\end{tabular}

Comparison of the figures presented in Table 2 by the use of Chi-square test, revealed that there is a significant association between the use of captopril in the change from the state of $\mathrm{LV}$ dysfunction to normal LV function $(\chi 2=5.8, p<0.05)$. Therefore, one may conclude that the early use of captopril, will be beneficial when compared to conventional therapy in maintaining a good state of LV function as well as on the long term basis.

Percentages shown on this table revealed that captopril is markedly better than conventional therapy in terms of LV dysfunction. In relation to the results obtained from Table 3, the statistical evidence of the benefit of captopril in improving $\mathrm{LV}$ function will be significantly emphasized.

\section{Discussion}

Captopril enhance cardiac performance in which it increases as a result of reduction in systemic vascular resistance and in left ventricular filling pressure [4]. Captopril has also antiarrhythmic action [5], and it has an action both on preload and afterload.

Improved survival with ACE inhibition in patients with myocardial infarction seems to be due to attenuation of infarct expansion and remodeling. Accordingly, changes, during the period at which these processes evolve. ACE inhibitors also used 
in order to prevent the ventricular dilatation which occurs early in the course of MI [6]. Thus treatment in appropriate patients should be started early within a few days, but not necessarily immediately after hospital admission [7]. So if we start ACE inhibitor therapy early you may actually prevent that activation - the rennin angiotensin system that maximally activated at three days - and therefore prevent any early activation that might occur in relation to the acute MI [8].

The mortality reduction with captopril was obtained against an already low mortality base line reflecting the haemodynamic stability of patients and their exposure to the best recommended treatment for the acute phase of the acute myocardial infarction. Therefore, reduction in mortality and left ventricular dysfunction is the net benefit of very early and systemic treatment.

The reduction in the mortality rate as well as the conversion from LV dysfunction to normal LV function, are the criteria used in order to evaluate the benefit of administrating captopril in many clinical trials. In our research, the mortality rate in the captopril group is $1 / 3$ the mortality rate in the conventional group. This result is in agreement with that found by Gruppo Italiano [9]. Converting LV dysfunction to normal LV function found in our study is also agreed with results obtained by Raya [10], Hargreaves [11], Simoons [12], and Pfeffer [13] and Collins [14]. From our results we conclude that the early and long term use of captopril will help in maintaining a good LV function as well as reducing mortality. So, once started captopril, the therapy can be continued indefinitely, unless side effects or contraindications develop.

\section{Conclusion}

This research work revealed that mortality rate in the group of patients used captopril was one third that of the other group of patients. Early and long term use of captopril will help maintaining a good LV function as well as reducing mortality rate.

\section{References}

1. James IM (1984) What's new in cardiovascular therapeutics. Medicine Digest 25: 48 .
2. David, Silverstein M (1981) Congestive cardiac failure-pathogenesis and treatment. Medicine Digest 7(8): 5-12.

3. Roger, Taylor R (1988) The pathophysiology of heart failure. Medicine Digest 14(7): 18-26.

4. Robertson J, Hodsman IS (1985) G.P. Captopril. Medicine Digest 11(7): $22-25$.

5. Pollak OJ (1988) Risk factors for atherosclerosis in proper perspective. Heart and Circulation 3(1): 18.

6. Peter S (1994) Introduction to the management of acute myocardial infarction. Brit J Cardiol 1(Suppl 1): 7-8.

7. Simoons ML (1994) Myocardial infarction: ACE-inhibitors for all? For every? The Lancet 344(8918): 279-280.

8. Collins R, Flather M, Peter S (1994) Discussion on presentation covering new development in management of MI. Brit J Cardiol 1(Suppl 1): 22-23.

9. Gruppo Italiano Io studio della soprawivenza nell'infarto miocardico (1994) GISSI-3: Effects of lisinopriland transdermal glyceryl trinitrate singly and together on 6-weeks mortality and ventricular function after acute myocardial infarction. The Lancet 343(8906): 1115-1121.

10. Raya TE, Gay RG, Aguirre M, Goldman S (1990) Importance of venodilatation in prevention of left ventricular dilatation after chronic large myocardial in rats: A comparison of captopril and hydralazine. Year Book of cardiology. (1990-6/95), Chapter 6, article 5.

11. Hargreaves AD, Kolletis T, Jacob AJ, Flint LL, Turnbull LW, et al. (1994) Early vasodilator in myocardial infarction: Appropriate for the majority and minority? Year Book of Cardiology Chapter 7, article 15.

12. Simoons ML (1994) Myocardial infarction: ACE-inhibitors for all? Forever? The Lancet 344(8918): 279-280.

13. Pfeffer MA (1994) The effect of captopril on mortality and morbidity in patients with left ventricular dysfunction after myocardial infarction: Results of the survival and ventricular enlargement trial. Year Book of Vascular Surgery Chapter 5, article 7.

14. Collins R, Flather M (1994) Fourth international study of infarct survival (ISIS-4). Brit J Cardiol 1(Suppl 1): 9-10.
This work is licensed under Creative Commons Attribution 4.0 License DOI: 10.19080/JOCCT.2018.11.555814 